\title{
Copper Accumulation in Rodent Brain Astrocytes: A Species Difference
}

\author{
Brendan Sullivan \\ Gregory Robison*, Ph.D. \\ Yulia Pushkar, Ph.D. \\ Department of Physics and Astronomy, Purdue University, \\ 525 Northwestern Ave., West Lafayette, IN 47907 \\ *Current Affiliation: Hanover College, Hanover IN
}

\author{
John K. Young, Ph.D. \\ Department of Anatomy, Howard University College of Medicine \\ 520 W St., NW, Washington DC 20059 \\ And \\ Kebreten F. Manaye \\ Department of Physiology, Howard University College of Medicine \\ 520 W St., NW, Washington DC 20059
}

Corresponding Author: Yulia Pushkar, Department of Physics and Astronomy, Purdue University, 525 Northwestern Ave., West Lafayette, IN 47907

Tel: (765) 496-3279; Fax: (765) 494-0706; ypushkar@purdue.edu. 


\begin{abstract}
Changes in $\mathrm{Cu}$ homeostasis have been implicated in multiple neurodegenerative diseases. Factors controlling and regulating the distribution of $\mathrm{Cu}$ in the brain remain largely unknown. We have previously reported that a sub-set of astrocytes in the subventricular zone (SVZ) contain Cu-rich aggregates. Here we expand previous studies with detailed X-ray fluorescent imaging (XRF) analysis of the additional brain areas of hippocampus (HP) and rostral migratory stream (RMS). We also use conventional DAB (3,3'-diaminobenzidine) staining which accesses both peroxidase and pseudoperoxidase activities. Both the HP and RMS support neurogenesis while the latter also serves as a migratory pathway for neuronal precursors. Some variations in neurogenic activities have been noticed between species (such as mice and rats). We report here that in rats, the HP, rostral migratory stream (RMS) and third ventricle contain glia which stain positively for DAB and contain copper-rich aggregates as measured by XRF. In contrast, mice hippocampi and RMS display neither DAB+ aggregates nor $\mathrm{Cu}$-rich accumulations via $\mathrm{XRF}$. DAB+ aggregates were not induced in the HP of mice transgenic for human amyloid precursor protein (APP) and presenilin, suggesting that accumulations positively stained for DAB are not directly caused by APP. These observed critical differences suggest different properties of the astrocytes in two species. Results suggest that the rat model may have important advantages over the mouse model for the study of hippocampal aging and neurodegeneration.
\end{abstract}

KEY WORDS: hippocampal aging, Alzheimer's, neurogenesis, Gomori-positive glia, X-ray fluorescence. 


\section{INTRODUCTION}

Owing to its extreme metabolic demands and the wide range of biochemistry facilitated by $\mathrm{Cu}$, the brain is capable of accumulating incredible amounts of $\mathrm{Cu}$ with concentrations second only to the liver [1]. The storage and uptake/export capacity of astrocytes have made them prime candidates for in vivo uptake of $\mathrm{Cu}$ into the brain where they are thought to act as "depots" for $\mathrm{Cu}$ sequestration in the brain $[2,3]$. Previous studies using energy dispersive spectroscopy (EDS) in neuroproliferative zones have reported metal-rich aggregates to be present in astrocytes, finding both $\mathrm{Fe}$ and $\mathrm{Cu}$ rich aggregates that exhibit peroxidase (or pseudoperoxidase) activity in rats [4-6]. Our more recent work with quantitative X-ray fluorescence (XRF) microscopy found $\mathrm{Cu}$-rich aggregates in slow-dividing astrocytes of the subventricular zone (SVZ) of both rats and mice where $\mathrm{Cu}$ concentrations increase with age [7]. Given the prevalence of transgenic mice in researching Cu-related diseases such as Menkes disease [8, 9], Alzheimer's disease [10], and prion disorders [11], it is important to determine if regions that form $\mathrm{Cu}$ aggregates in rats also do so in mice to further validate these models.

Using X-ray fluorescence microscopy, it is demonstrated that the rat but not mouse dentate gyrus and rostral migratory stream (RMS) - additional neuroproliferative zone in adults - contain $\mathrm{Cu}$-rich aggregates. Both rats and mice exhibit $\mathrm{Cu}$ aggregates in regions of the habenula and hypothalamus adjacent to the third ventricle. These results are mirrored by DAB (3,3'-diaminobenzidine) staining which accesses peroxidase or pseudoperoxidase activity. DAB staining, however, lacks elemental specificity and has generated some ambiguity as to whether $\mathrm{Cu}$ or Fe aggregates were responsible for $\mathrm{DAB}+$ staining in these regions. Using combined $\mathrm{DAB}$ and $\mathrm{XRF}$ imaging, we report that both $\mathrm{Cu}$ and Fe aggregates are responsible for a subset of $\mathrm{DAB}+$ staining in these regions.

The results presented here show that $\mathrm{Cu}$ is distributed differently in rat and mouse brains and that subsets of astrocytes display different $\mathrm{Cu}$ accumulation properties. While the role of these $\mathrm{Cu}$ 
aggregates remains elusive, the present work serves as a baseline to characterize species differences.

Given the importance of astrocytes in metal distribution and brain function, detected differences may have important consequences in choosing rodent models for studying neurogenesis, aging, metal homeostasis, and neurodegeneration.

\section{MATERIALS AND METHODS}

Animals. For XRF, 7-8 weeks old Male Sprague-Dawley rats from Harlan Laboratories were purchased and were housed in a temperature-controlled, 12/12 light/dark room, and allowed free access to pelleted rat chow (Purina rodent chow 5001) and distilled, deionized water. Three nine-week old 129S1/SvImJ mice were purchased from the Jackson Laboratory, three CD-1 mice from Charles River Laboratories and three female C57BL/6J×SvImJ from Jackson Laboratory. At the age given in Table 1, the animals were sacrificed and the brains were dissected and frozen directly on dry ice. For histochemical labeling, 4 male and 2 female mice (C57bl/6j strain) about 16 weeks old and SpragueDawley rats were used initially. To confirm that observed differences in mice were not strain-specific, brains from four 7-month old mice of the C57bl strain were examined. Two of these mice were wild type, control mice, and two additional mice were transgenic for human amyloid precursor protein and presenilin-1 [12] as amyloid precursor protein exerts damaging, oxidative stress upon astrocyte mitochondria and thus could possibly contribute to the development of the astrocytic pathology in the hippocampus [13]. Additionally, brains from six 8-month old retired breeder males of the albino CD-1 strain were obtained. All experiments complied with appropriate institutional animal use and care committees.

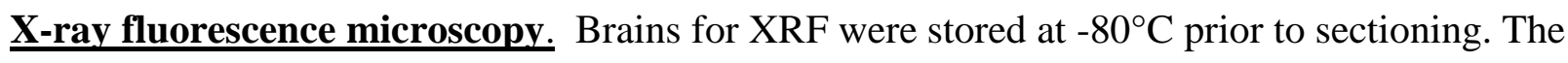
night before sectioning, brains were warmed to $-20^{\circ} \mathrm{C}$ and ultimately to $-12^{\circ} \mathrm{C}$ for sectioning. With the exception of $\mathrm{DAB}+\mathrm{XRF}$ co-localization, brains were not chemically treated in any way (e.g. fixation or 
perfusion) to avoid metal redistribution. To identify planes containing the SVZ and HP, cresyl violet stains were performed on sections adjacent to regions before and after those taken for imaging. Planes taken contained the SVZ or HP (coronal sections) or the SVZ, HP, and RMS (sagittal sections.) Sections for XRF imaging were thawed onto $4 \mu \mathrm{m}$ thick polypropylene film stretched on frames and frozen immediately. Adjacent sections were taken on glass slides for conventional microscopy. All samples were stored at $-80^{\circ} \mathrm{C}$ prior to imaging.

Imaging was carried out at the Advanced Photon Source at Argonne National Laboratory (Argonne, IL) using beamlines 2-ID-D, 2-ID-E, 8-BM, BioCAT (18-ID) the Nanoprobe (26-ID), the Bionanoprobe (21-ID) and from beamline 10.3.2 at the Advanced Light Source (Berkeley, CA). Fluorescence spectra were recorded using a silicon drift detector and the obtained spectra were either fitted on a pixel-by-pixel basis by the MAPS program [14] or recorded as counts in the $\mathrm{Cu}$ energy range from a single channel analyzer. For absolute quantitation, spectra were compared to spectra recorded from either the NBS1832/1833 standards (NIST, Gaithersburg, MD) or the AXO thin film standard (AXO Dresden GmbH, Dresden, Germany) under the same experimental conditions.

As XRF illuminates an entire column through the sample thickness, the resulting measurement after comparing to a thin film has units of mass per area. It is straightforward to convert this mass into moles. The sample thickness is known from section preparation (5-30 $\mu \mathrm{m}$ in all work here), and so the reported area represents a volume obtained by multiplying by the thickness. It is therefore possible, and often convenient, to reported metals as concentrations.

Quantitation of SVZ and RMS Cu concentrations. Quantification of $\mathrm{Cu}$ concentration in the narrow regions in the SVZ and RMS are non-trivial as the resulting concentration is dependent to some extent on the experimenter's choice of ROI. To mitigate this dependence, we have analyzed the mean concentration as in the past for rats from images of the same spatial resolution [15]. Briefly, a line is 
drawn along the ventricle wall by the experimenter and the ROI for quantitation is defined by expanding that line by 3 pixels $(75 \mu \mathrm{m})$ in each cardinal direction. While this means that the reported concentration will be lower than the true concentration, defining the ROI in this way defines a consistent means for analysis with as little variability due to ROI selection as possible.

Histochemistry for DAB+ astrocytic aggregates. Animals were given an overdose of pentobarbital and perfused through the heart with $0.9 \%$ saline followed by $4 \%$ formalin in $0.2 \mathrm{M}$ phosphate buffer. Brains were removed, fixed for an additional 2 days, and transferred to $20 \%$ sucrosephosphate buffer for cryoprotection. Brains were blocked and small chunks were cut from the cortex at different places for each brain for purposes of subsequent identification of brain sections. Then, serial $35 \mu \mathrm{m}$ thick frozen sections of each brain were cut on a freezing microtome. Sections were collected beginning at the level of the anterior habenula or anterior hypothalamus and ending at the level of the posterior hypothalamus and where the hippocampus begins to descend ventrally into the temporal lobe.

To identify $\mathrm{DAB}+$ astrocytes, every other section from each brain was placed into a mixture of diaminobenzidine (DAB) and hydrogen peroxide for $30 \mathrm{~min}$. (25 mg of DAB were added to $50 \mathrm{ml}$ of

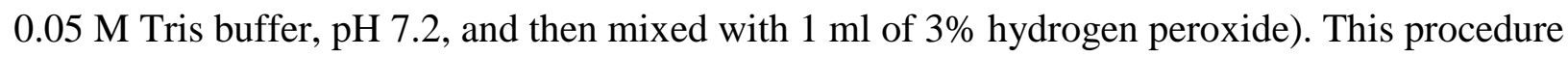
reliably detects metal-containing aggregates in rat brains [16-18]. Following the staining procedure, sections were washed in 3 changes of phosphate buffer. Sections were mounted on subbed slides. Selected sections were lightly counterstained with $0.1 \%$ toluidine blue in acetate buffer, and then dehydrated and coverslipped. The DAB+ aggregates surrounding astrocyte nuclei were examined using bright-field and dark-field microscopy.

For the 8 month old CD-1 mice, $100 \mu \mathrm{m}$ thick sections of one rat and one mouse brain were prepared using a vibratome and were stained to demonstrate immunoreactivity for glial fibrillary acidic protein (GFAP). Briefly, free floating sections were exposed to a rabbit antibody to bovine GFAP (Dako 
Corp., Carpenteria, CA), diluted 1:2500 in 3\% normal goat serum-phosphate buffered saline, washed, incubated with biotinylated goat-antirabbit IgG (Vectastain ABC kit, Vector Laboratories, Burlingame, CA), and exposed to $0.3 \%$ hydrogen peroxide for 30 minutes to deplete endogenous sources of peroxidase activity. Sections were exposed to $\mathrm{ABC}$ reagent for 60 minutes, followed by incubation in $0.05 \%$ DAB in Tris buffer to develop the color reaction for GFAP staining. Sections were then dehydrated, embedded in JV-4 methacrylate resin between plastic cover slips, and then glued onto methacrylate blocks with cyanoacrylate glue [18]. Semi-thin sections ( $2 \mu \mathrm{m}$ thick) were prepared using dry glass knives. Sections were placed onto subbed slides and counterstained with toluidine blue to illustrate the features of aggregate-containing astrocytes.

Co-localization of XRF and Histochemistry. A $30 \mu \mathrm{m}$ thick section of a C57BL/6J mouse was incubated in a DAB solution for 30 minutes followed by $3 \times 5$ minute rinses with phosphate buffered saline. To remove excess $\mathrm{P}, \mathrm{K}$, and $\mathrm{Na}$ from the sample for XRF mapping, the sample was quickly rinsed twice with distilled and deionized water. X-ray maps were recorded at beamline 2-ID-D at the Advanced Photon Source with a beam size of $250 \times 250 \mathrm{~nm}^{2}$ and an incident flux of $4 \times 10^{9}$ photons per second at $10 \mathrm{keV}$. Immediately after X-ray measurements, the sample was imaged optically. Colocalization was carried out using the landmark correspondence [19] by transforming the optical image to match features on the X-ray maps allowing translation, isotropic scaling, and rotations (i.e. under a similarity transform).

Fe and $\mathrm{Cu}$ Co-localization Statistics. To determine if $\mathrm{Fe}$ and $\mathrm{Cu}$ co-localize in individual clusters, images with 30 or $40 \mathrm{~nm}$ resolution were thresholded at $17.9 \mathrm{mM}$ for Fe or $31.5 \mathrm{mM} \mathrm{for} \mathrm{Cu}$ (corresponding to 0.5 and $1.0 \mu \mathrm{g} \mathrm{cm}^{-2}$ in $5 \mu \mathrm{m}$ thick sections). Particles were then defined by requiring at least one of the surrounding eight pixels to be above the threshold. In other words, if two connected sets of pixels have no connecting pixels they are treated as two separate clusters. To reduce any 
fluctuations due to noise, only clusters with 5 or more pixels were considered in analysis. For clarity, an example of particle detection is shown in Fig. 5B where arrows indicate particles used for analysis. For each cluster, Pearson's correlation coefficient, $r$, was computed using all pixels in the cluster. A total of $n=17 \mathrm{Cu}$ clusters and $n=22 \mathrm{Fe}$ clusters were included in analysis from two 9-week old 129S1/SvImJ mice. A dotplot of correlations along with a boxplot of the distribution is given in Fig. 5C.

\section{RESULTS}

In the dentate gyrus of the hippocampus, rats showed DAB+ staining (Fig. 1C) and $\mathrm{Cu}$ aggregates were detected using XRF (Fig. 1D). Mice of all ages and strains examined did not exhibit $\mathrm{DAB}+$ straining or Cu-rich hippocampal accumulations (Fig. 1A, B). Similarly, the hippocampi of mice transgenic for human amyloid precursor protein and presenilin-1 failed to show any DAB+ accumulations (data not shown). Both rats and mice exhibit Fe aggregates in the granular layer of the hippocampus and a few Fe aggregates in the dentate gyrus.

We have previously reported that both rats and mice display intracellular $\mathrm{Cu}$-rich accumulations in the SVZ [7] and rats have elevated Cu along the RMS ([15]; Fig. 2 B,E), it remained undetermined if the increased $\mathrm{Cu}$ concentration along the RMS was also due to intracellular $\mathrm{Cu}$-rich aggregates. $\mathrm{XRF}$ imaging with $1.5 \mu \mathrm{m}$ resolution (Fig. 2C) demonstrates that the elevated $\mathrm{Cu}$ in the RMS is due primarily to localized $\mathrm{Cu}$ aggregates. The RMS of mice, by contrast, does not possess $\mathrm{Cu}$ aggregates (Fig. 2D). $\mathrm{Cu}$ concentrations in four different brain areas of 12 week old Sprague-Dawley rats and CD-1 mice are given in Table 2. It is clear that the mouse RMS has considerably lower concentrations than the SVZ while in rats the $\mathrm{Cu}$ concentrations in the rat RMS and $\mathrm{SVZ}$ are comparable. The presence of $\mathrm{Cu}$-rich aggregates is mirrored by the presence of DAB+ aggregates in the RMS (Fig. 2A,D)

Sections from both rats and mice of all examined ages and strains showed numerous clusters of $\mathrm{DAB}+$ aggregates within the hypothalamus and the habenula in regions near the third ventricle (Fig. 3B- 
E). The distributions of DAB+ astrocytes were very similar in these areas between rats and mice. These findings confirm previously published studies [20,21]. Similarly, both rats and mice have $\mathrm{Cu}$ aggregates along the third ventricle in both the habenula (Fig. 3F-H) and hypothalamus (data not shown). At higher magnification, sections of rat and mouse brain revealed the presence of pale, ovoid astrocyte nuclei surrounded by a cloud of dark-staining with DAB+ aggregates (Fig. 3A).

To analyze the correspondence of the DAB stain with metal distributions we conducted DAB staining and XRF analysis of the SVZ area of rat brain. The SVZ was selected as it possesses significant $\mathrm{Cu}$ accumulations. Co-localization of the optical DAB image with $\mathrm{Fe}$ and $\mathrm{Cu}$ distributions detected by XRF are shown in Fig. 4C. It should be noted that the staining process likely modifies the metal distribution resulting in the loss of mobile metal ions. $\mathrm{DAB}+$ staining was observed in several situations with respect to metal presence. Some stained spots are observed in the absence of metal aggregates. Such staining would have either a non-metal origin of protein based peroxidase activity or a very dilute metal origin for peroxidase or pseudo peroxidase activity. It is also possible that $\mathrm{DAB}+$ stains that do not co-localize with metal were a result of metals that had been washed away during the staining process. More commonly in the SVZ, DAB+ staining co-localizes very well with $\mathrm{Cu}, \mathrm{Fe}$, or $\mathrm{Cu}$ and $\mathrm{Fe}$ aggregates, rendering the metal accumulation a likely source of pseudo peroxidase activity.

Imaging of the $\mathrm{Cu}$-rich cell on fresh, unstained sample (i.e., where the distribution of elements is not affected by any staining procedure) in the subventricular zone with $30 \mathrm{~nm}$ resolution revealed two types of metal accumulations (Fig. 5). The first, and most numerous, were Cu-rich spheroids. Spheroids are often found near each other and are approximately $1 \mu \mathrm{m}$ in diameter (e.g. Fig. 5D). When non-spherical accumulations are present (e.g. Fig. 5A), this is likely the result of overlapping spheroids through the $5 \mu \mathrm{m}$ sample depth as the intensity profile is well described as a series of singly-peaked distributions. Also present, and distinct from $\mathrm{Cu}$ aggregates, are ovoid-shaped Fe-rich aggregates of 
comparable size (Fig. 5A). In Cu-rich aggregates, the mean $\mathrm{Cu}$ concentration is $113.0 \mathrm{mM}$ with a mean Fe concentration of $3.6 \mathrm{mM}$. In Fe-rich aggregates, the mean $\mathrm{Cu}$ concentration is $2.5 \mathrm{mM}$ while the mean Fe concentration is $27.2 \mathrm{mM}$.

To determine if $\mathrm{Fe}$ and $\mathrm{Cu}$ co-localize in aggregates, Pearson's overlap coefficient was calculated for $\mathrm{Fe}$ and $\mathrm{Cu}$ from XRF maps with $30 \mathrm{~nm}$ or $40 \mathrm{~nm}$ resolution on a cluster-by-cluster basis (Fig. 5C). In Cu-rich clusters, the average correlation was found to be $r=0.03$ with a median of $r=$ 0.03. In Fe rich clusters ( $n=22$ identified clusters), the mean was $r=0.01$ with a median $r=0.06$. These data show that $\mathrm{Fe}$ and $\mathrm{Cu}$ do not co-localize in unstained sections, with rare exception (e.g. Fig. 5D).

\section{DISCUSSION}

XRF microscopy with sub-micron resolution was able to show the presence of $\mathrm{Cu}$-rich aggregates in the dentate gyrus and rostral migratory stream in the rat brain which were not detected in mice. These results were mirrored by DAB staining (Figs. 1-3). While to the best of our knowledge there are no studies that directly compare the rat and mouse RMS, differences in the hippocampus have been characterized and so it serves as a good starting point for rationalization. One plausible explanation is that mice show reduced hippocampal neurogenesis and circuital integration in comparison with rats. For example, newly born neurons in the dentate gyrus are three-fold more numerous in adult rats as compared to adult mice, and are ten times more likely to be recruited into learning circuits [22]. A diminished number of neurogenic astrocytes in mice, as well as reduced oxidative stress, may well be the explanation for the species difference we observed.

Other species differences in the rat and mouse hippocampus have been reported. In the mouse, dentate gyrus neurons are immunoreactive for calretinin, but in the human and rat these neurons fail to 
stain for this calcium-binding protein [23, 24]. Also, cholecystokinin immunoreactivity can be found in the mouse dentate gyrus, but not in the rat. Finally, dentate gyrus neurons of the mouse fails to stain for calcitonin gene-related peptide, but those of the rat show prominent staining [23].

Hippocampal neurodegenerative processes could relate to the copper accumulation and mitochondrial degeneration in neurogenic astrocytes. Copper plays a major role in the processing of beta-amyloid, a molecule central to the neurodegeneration seen in Alzheimer's disease [25, 26]. Also, the protection of neurons from toxic effects of amyloid involves astrocytic mitochondria, and could be compromised if astrocytic mitochondria are damaged [27]. A number of features of neurogenic, coppercontaining astrocytes suggest that they may explain the special vulnerability of the hippocampus to neurodegenerative processes in Alzheimer's disease [28]. The differences between mouse and rat hippocampus, reported and reviewed here, suggest that the rat may be a more suitable model for studying age-related changes in neurogenesis and neurodegeneration than the mouse $[29,30]$.

Amyloid itself has been found to damage mitochondria within astrocytes [13]. However, the failure of the elevated production of APP in the transgenic mice studied here to provoke the appearance of $\mathrm{DAB}+$ staining within the hippocampus suggests that plaque deposition per se is not a precipitating factor for the development of copper deposition and mitochondrial damage.

There are conflicting claims in the literature that peroxidase activity exhibiting (DAB+) aggregates derive from Fe complexed with porphyrins $[4,31,32]$ while other reports demonstrate that accumulations are $\mathrm{Cu}$-rich [5, 33] as measured by energy dispersive spectroscopy (EDS). Similarly, our XRF data with 30-40 $\mathrm{nm}$ resolution characterizing untreated rats show $\mathrm{Cu}$-rich aggregates and a separate set of Fe-rich aggregates (Fig. 5A). Co-localization analysis concludes that $\mathrm{Cu}$ aggregates do not contain Fe and vice-versa. All of the spherical metal aggregates we observed, however, were primarily composed of $\mathrm{Cu}$ and do not co-localize with $\mathrm{Fe}$. Furthermore, both Fe-rich and $\mathrm{Cu}$-rich aggregates can 
co-localize with DAB staining (Fig. 4C), suggesting they are each capable of peroxidase or pseudo peroxidase activity.

As for the binding proteins, in Fe aggregates both heme $[4,5]$ and non-heme Fe coordination have been proposed. For instance, similar Fe aggregates were recently examined in neurons and were suspected to be siderosomes while hold Fe as ferric hydroxide [34]; ferritin has also been proposed [5]. As both heme and non-heme Fe are capable non-enzymatic peroxidase activity [35, 36], the present data is unable to determine a source. With respect to the source of Cu DAB staining, our previous work has shown that $\mathrm{Cu}$ is present as $\mathrm{Cu}(\mathrm{I})$ and as a $\mathrm{Cu}_{\mathrm{x}} \mathrm{S}_{\mathrm{y}}$ cluster with a $[\mathrm{S}] /[\mathrm{Cu}]$ ratio consistent with that of $\mathrm{Cu}-$

metallothionein [7]. As metallothionein has an established role in hydrogen peroxide protection [37], it seems plausible that an aggregate composed of metallothionein or a metallothionein-like protein could exhibit peroxidase activity.

\section{ACKOWLEDGEMENTS}

We gratefully acknowledge the assistance of Dr. Donald Ingram in providing brain tissue from mice transgenic for human APP and presenilin-1. We acknowledge the Zheng Laboratory at Purdue University for help with animal treatment and sacrifice of XRF imaged animals. We are grateful to Dr. David Vine, Dr. Barry Lai, Dr. Raul Barrea, Dr. Lydia Finney, Evan Maxey, Dr. Volker Rose, and Dr. Jungjing Deng for the help operating beamlines at the Advanced Photon Source. This work was funding by NIH 2U54NS039407-10 and NIH R01 ES008146-14. Use of the Advanced Photon Source, an Office of Science User Facility operated for the U.S. Department of Energy (DOE) Office of Science by Argonne National Laboratory, was supported by the U.S. DOE under contract number DE-AC0206CH11357. The Biophysics Collaborative Access Team is a National Institutes of Health (NIH)supported Research Center RR-08630. The operations of the ALS at LBNL are supported by the 
Director, Office of Sciences, Office of Basic Energy Sciences, U.S. Department of Energy under contract number DE-AC02-05CH11231. 


\section{References}

1. Bush, A.I., Metals and neuroscience. Curr. Opin. Chem. Biol. 4 (2000) 184-191.

2. Scheiber, I.F. and R. Dringen, Astrocyte functions in the copper homeostasis of the brain. Neurochem. Int. 62 (2013) 556-565.

3. Tiffany-Castiglioni, E. and Y. Qian, Astroglia as metal depots: molecular mechanisms for metal accumulation, storage and release. Neurotoxicology. 22 (2001) 577-592.

4. Brawer, J.R., et al., The origin and composition of peroxidase-positive granules in cysteaminetreated astrocytes in culture. Brain Res. 633 (1994) 9-20.

5. $\quad$ Brawer, J.R., et al., Composition of Gomori-positive inclusions in astrocytes of the hypothalamic arcuate nucleus. The Anatomical Record. 240 (1994) 407-415.

6. Schipper, H.M. and A. Mateescu-Cantuniari, Identification of peroxidase-positive astrocytes by combined histochemical and immunolabeling techniques in situ and in cell culture. J. Histochem. Cytochem. 39 (1991) 1009-1016.

7. Pushkar, Y., et al., Aging results in copper accumulations in glial fibrillary acidic proteinpositive cells in the subventricular zone. Aging Cell. 12 (2013) 823-32.

8. Hunt, D., Primary defect in copper transport underlies mottled mutants in the mouse. Nature. 249 (1974) 852-854.

9. Levinson, B., et al., The mottled gene is the mouse homologue of the Menkes disease gene. Nature genetics. 6 (1994) 369-373.

10. Games, D., et al., Alzheimer-type neuropathology in transgenic mice overexpressing $V 717 F \quad \beta$ amyloid precursor protein. (1995).

11. Telling, G.C., et al., Transmission of Creutzfeldt-Jakob disease from humans to transgenic mice expressing chimeric human-mouse prion protein. Proceedings of the National Academy of Sciences. 91 (1994) 9936-9940.

12. O’Neil, J.N., et al., Catecholaminergic neuronal loss in locus coeruleus of aged female dtg APP/PS1 mice. Journal of chemical neuroanatomy. 34 (2007) 102-107.

13. Abramov, A.Y., L. Canevari, and M.R. Duchen, $\beta$-amyloid peptides induce mitochondrial dysfunction and oxidative stress in astrocytes and death of neurons through activation of NADPH oxidase. The Journal of neuroscience. 24 (2004) 565-575.

14. Vogt, S., MAPS: A set of software tools for analysis and visualization of $3 D X$-ray fluorescence data sets. J. Phys. IV. 104 (2003) 635-638.

15. Sullivan, B., et al., Mn exposure does not affect neurogenesis. Toxicological Sciences. Manuscript Accepted (2016).

16. Keefer, D.A. and J.F. Christ, Distribution of endogenous diaminobenzidine-staining cells in the normal rat brain. Brain Res. 116 (1976) 312-316.

17. Justino, L., et al., Long-term effects of cysteamine on cognitive and locomotor behavior in rats: relationship to hippocampal glial pathology and somatostatin levels. Brain Res. 761 (1997) 127134.

18. Young, J., J. McKenzie, and J. Baker, Association of iron-containing astrocytes with dopaminergic neurons of the arcuate nucleus. Journal of neuroscience research. 25 (1990) 204213.

19. Saalfeld, S. and P. Tomancák. Automatic landmark correspondence detection for ImageJ. in Proceedings of the ImageJ User and Developer Conference. 2008. 
20. Terr, L. and L. Weiner, Structural and cytochemical changes in astrocytes from the brain periventricular zone of the copper-deficient blotchy mouse. The Anatomical Record. 205 (1983) 347-353.

21. Young, J.K., Immunoreactivity for diazepam binding inhibitor in Gomori-positive astrocytes. Regulatory peptides. 50 (1994) 159-165.

22. Snyder, J.S., et al., Adult-born hippocampal neurons are more numerous, faster maturing, and more involved in behavior in rats than in mice. The Journal of Neuroscience. 29 (2009) 1448414495.

23. Sakurai, O. and T. Kosaka, Nonprincipal neurons and CA2 pyramidal cells, but not mossy cells are immunoreactive for calcitonin gene-related peptide in the mouse hippocampus. Brain Res. 1186 (2007) 129-143.

24. Seress, L., et al., Calretinin expression in hilar mossy cells of the hippocampal dentate gyrus of nonhuman primates and humans. Hippocampus. 18 (2008) 425-434.

25. Hou, P., et al., The role of copper and the copper-related protein CUTA in mediating APP processing and Aß generation. Neurobiology of aging. 36 (2015) 1310-1315.

26. Zheng, W., et al., Divalent metal transporter 1 is involved in amyloid precursor protein processing and A generation. The FASEB Journal. 23 (2009) 4207-4217.

27. Aguirre-Rueda, D., et al., Astrocytes Protect Neurons from AB1-42 Peptide-Induced Neurotoxicity Increasing TFAM and PGC-1 and Decreasing PPAR- $\gamma$ and SIRT-1. International journal of medical sciences. 12 (2015) 48.

28. Young, J., Hippocampal astrocytes and Alzheimer's Disease. J. Prevent. Alz. Dis. 1 (2014) 124128.

29. Flood, D.G., et al., A transgenic rat model of Alzheimer's disease with extracellular A $\beta$ deposition. Neurobiology of aging. 30 (2009) 1078-1090.

30. Liu, L., et al., A transgenic rat that develops Alzheimer's disease-like amyloid pathology, deficits in synaptic plasticity and cognitive impairment. Neurobiol. Dis. 31 (2008) 46-57.

31. Goldgefter, L., A. Schejter, and D. Gill, Structural and microspectrofluorometric studies on glial cells from the periventricular and arcuate nuclei of the rat hypothalamus. Cell and tissue research. 211 (1980) 503-510.

32. McLaren, J., J. Brawer, and H. Schipper, Iron content correlates with peroxidase activity in cysteamine-induced astroglial organelles. J. Histochem. Cytochem. 40 (1992) 1887-1897.

33. Szerdahelyi, P. and P. Kasa, Histochemical demonstration of copper in normal rat brain and spinal cord - evidence of localization in glial cells. Histochemistry. 85 (1986) 341-347.

34. Colvin, R.A., et al., Understanding metal homeostasis in primary cultured neurons. Studies using single neuron subcellular and quantitative metallomics. Metallomics, (2015).

35. Poulos, T.L. and J. Kraut, The stereochemistry of peroxidase catalysis. J. Biol. Chem. 255 (1980) 8199-8205.

36. Jin, S., et al., X-ray crystal structures of reduced rubrerythrin and its azide adduct: a structurebased mechanism for a non-heme diiron peroxidase. J. Am. Chem. Soc. 124 (2002) 9845-9855.

37. Quesada, A.R., et al., Direct reaction of $\mathrm{H} 2 \mathrm{O} 2$ with sulfhydryl groups in HL-60 cells: zincmetallothionein and other sites. Arch. Biochem. Biophys. 334 (1996) 241-250. 


\section{Figures and Figure Captions}
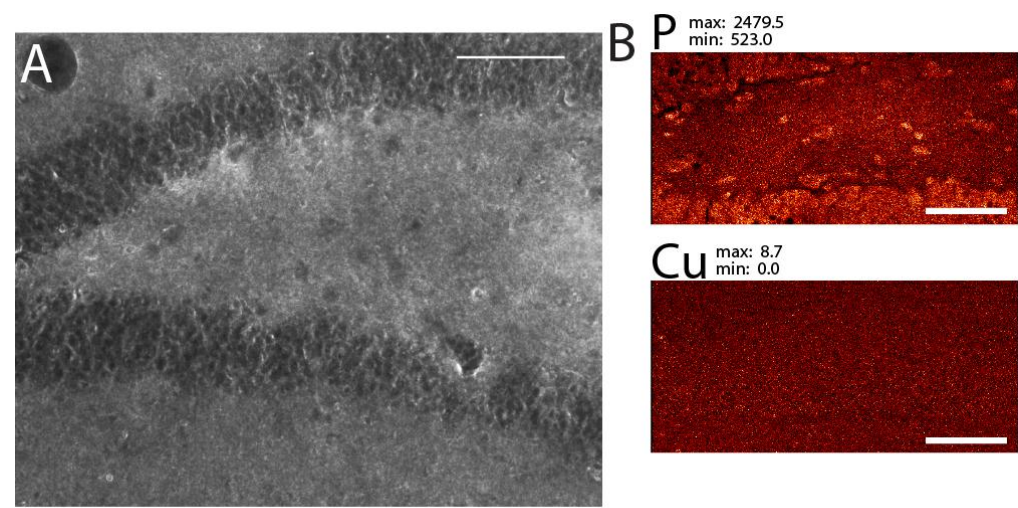

$\mathrm{Fe} \underset{\min }{\max 3.2 .2}$

$\mathrm{Cu} / \mathrm{Fe} / \mathrm{P}$
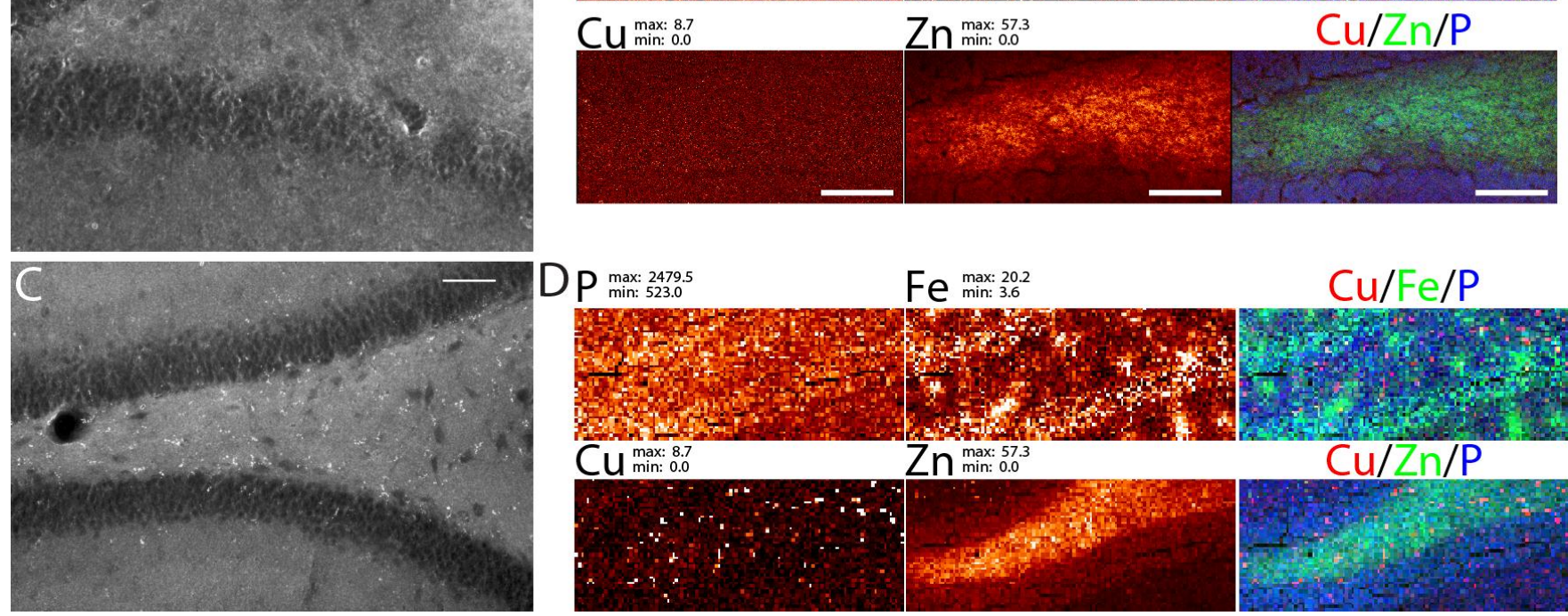

$\mathrm{Cu} / \mathrm{Zn} / \mathrm{P}$

Figure 1. Distribution of $\mathrm{Cu}$ aggregates in the hippocampus. A. Dark field micrograph of a DABstained mouse section shows no aggregates. B. X-ray fluorescence maps of a mouse hippocampus also shows no $\mathrm{Cu}$ accumulations, confirming that the $\mathrm{Cu}$ aggregates present in rats and in the $\mathrm{SVZ}$ of both species are not present. C. Dark field micrograph of a DAB-stained rat section reveals positivelystained aggregates in the dentate gyrus. D. Similarly, elemental maps show dense $\mathrm{Cu}$ aggregates in dentate gyrus of the rat hippocampus. Scale bars: $100 \mu \mathrm{m}$. Display ranges are in $\mathrm{mM}$ through a $10 \mu \mathrm{m}$ thick section. 

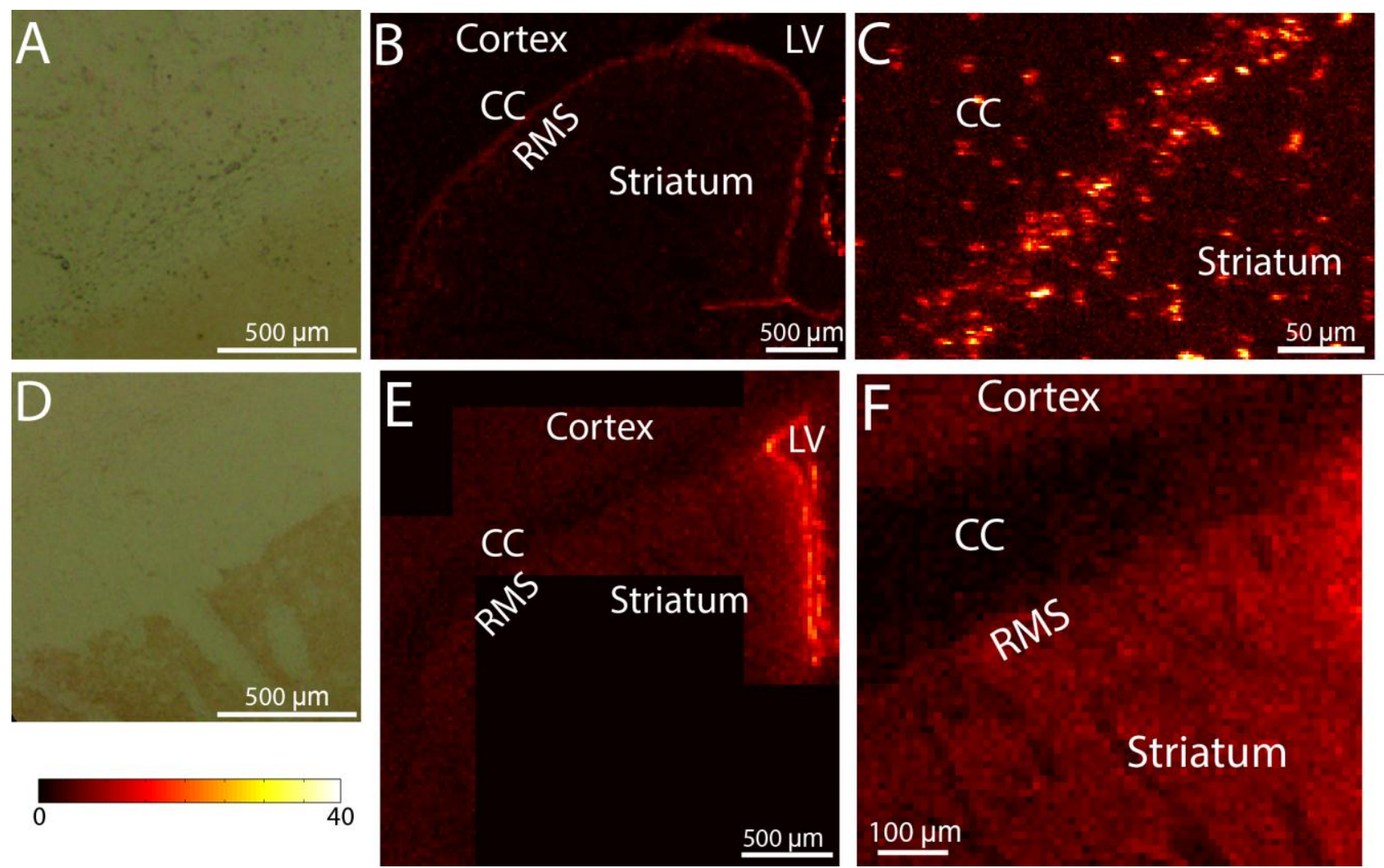

\section{Cortex}

$\mathrm{CC}$

\section{Striatum}

Figure 2. A. DAB stained RMS in a rat shows small DAB+ aggregates. B. Cu XRF map of a rat SVZ and RMS showing that $\mathrm{Cu}$ concentrations in the RMS are comparable to that of the SVZ. C. Higher magnification $\mathrm{Cu} \mathrm{XRF}$ map demonstrating that the majority of $\mathrm{Cu}$ is derived from localized $\mathrm{Cu}$ aggregates. D. DAB staining of a mouse RMS showing that there are no DAB+ aggregates. E. Cu XRF map of a mouse SVZ and RMS. F. Higher magnification Cu XRF map of the RMS. In contrast to rats, mice RMS does not have $\mathrm{Cu}$-rich aggregates in the RMS. 

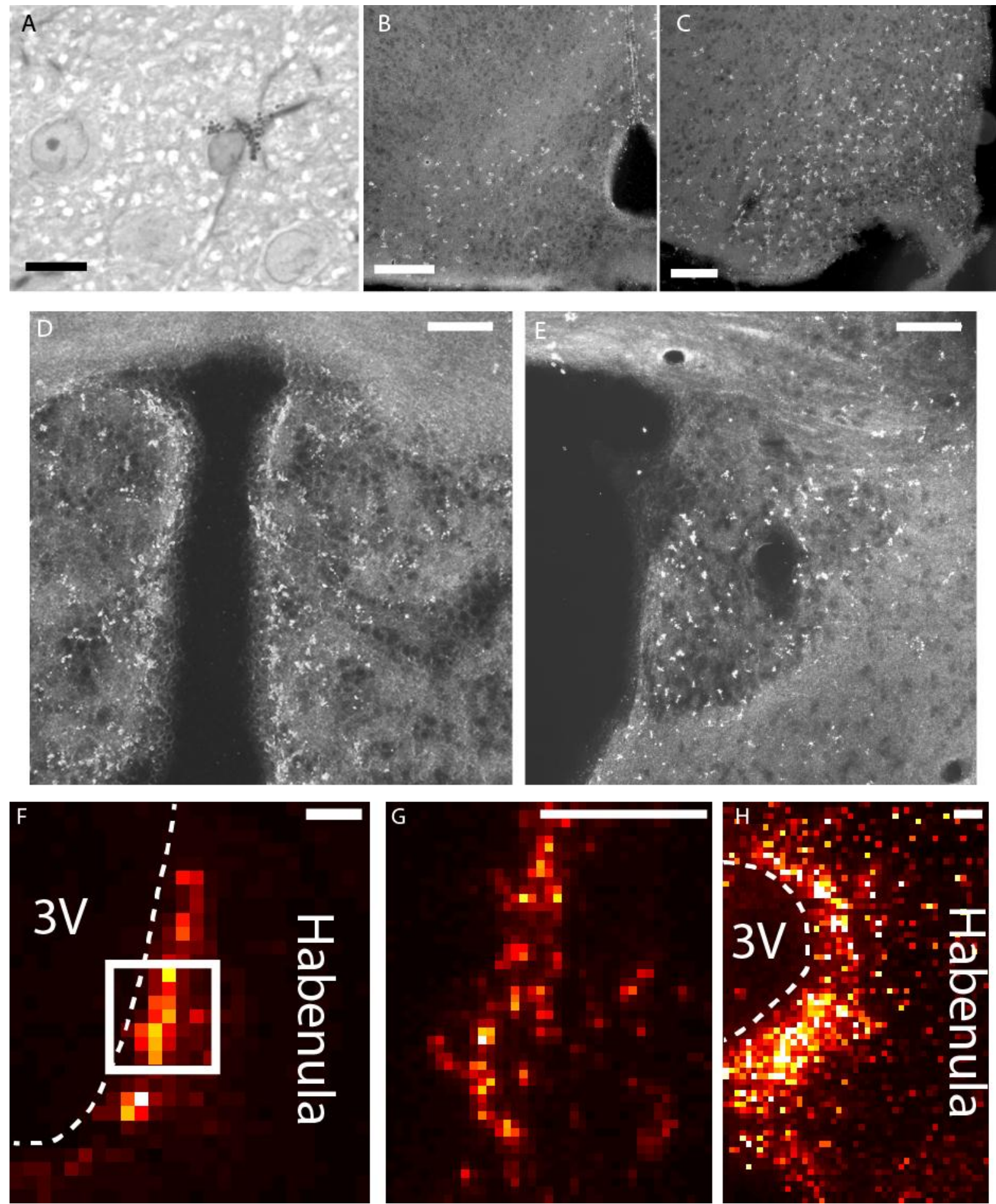

Figure 3. A. High magnification view of a DAB-stained astrocyte, stained for GFAP using immunocytochemistry and counterstained with toluidine blue. An astrocyte possesses a cloud of $\mathrm{DAB}+$ aggregates and GFAP-immunoreactive filaments surrounding an ovoid, pale-staining nucleus. Scale bar: $10 \mu \mathrm{m}$. B-E. Dark field micrographs for DAB-stained Gomori-positive astrocytes in frozen sections. DAB+ aggregates can be found in the arcuate nucleus and periventricular zone in the hypothalamus of both mice (B) and rats (C). The habenula in both mice (D) and rats (E) also 
exhibit similar distributions of DAB staining. DAB stain appears bright under Dark field microscopy. F-H. $\mathrm{Cu}$ XRF maps of mouse $(\mathrm{F}, \mathrm{G})$ and rat $(\mathrm{H})$ habenulae demonstrating elevated $\mathrm{Cu}$ along the third ventricle. The box in F outlines the region imaged in G. Scale bars: $100 \mu \mathrm{m}$. 

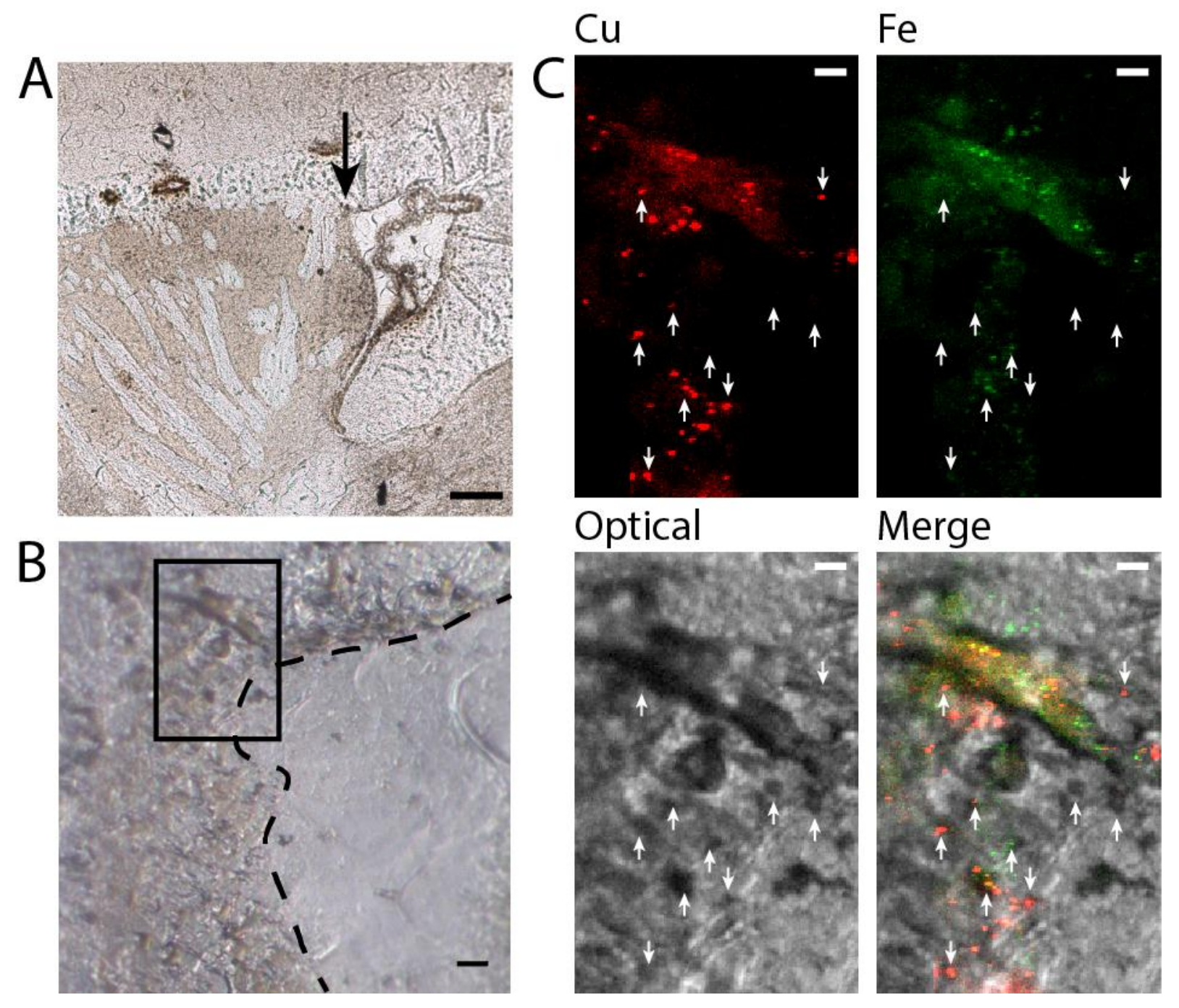

Figure 4: A. Low-magnification micrograph of the DAB-stained section. The arrow points to the rostral corner of the ventricle, which was imaged. Scale bar: $100 \mu \mathrm{m}$. B. Untransformed optical micrograph of the rostral corner. The dashed line indicates the ventricle wall and the box outlines the area imaged with X-ray microscopy. Scale bar: $10 \mu \mathrm{m}$. C. Co-localization of $\mathrm{Cu}, \mathrm{Fe}$, and optical images showing that $\mathrm{DAB}+$ aggregates often, but not always, co-localize with metal aggregates. Note that the optical image has been transformed as described in the methods section. Scale bar: $5 \mu \mathrm{m}$. 


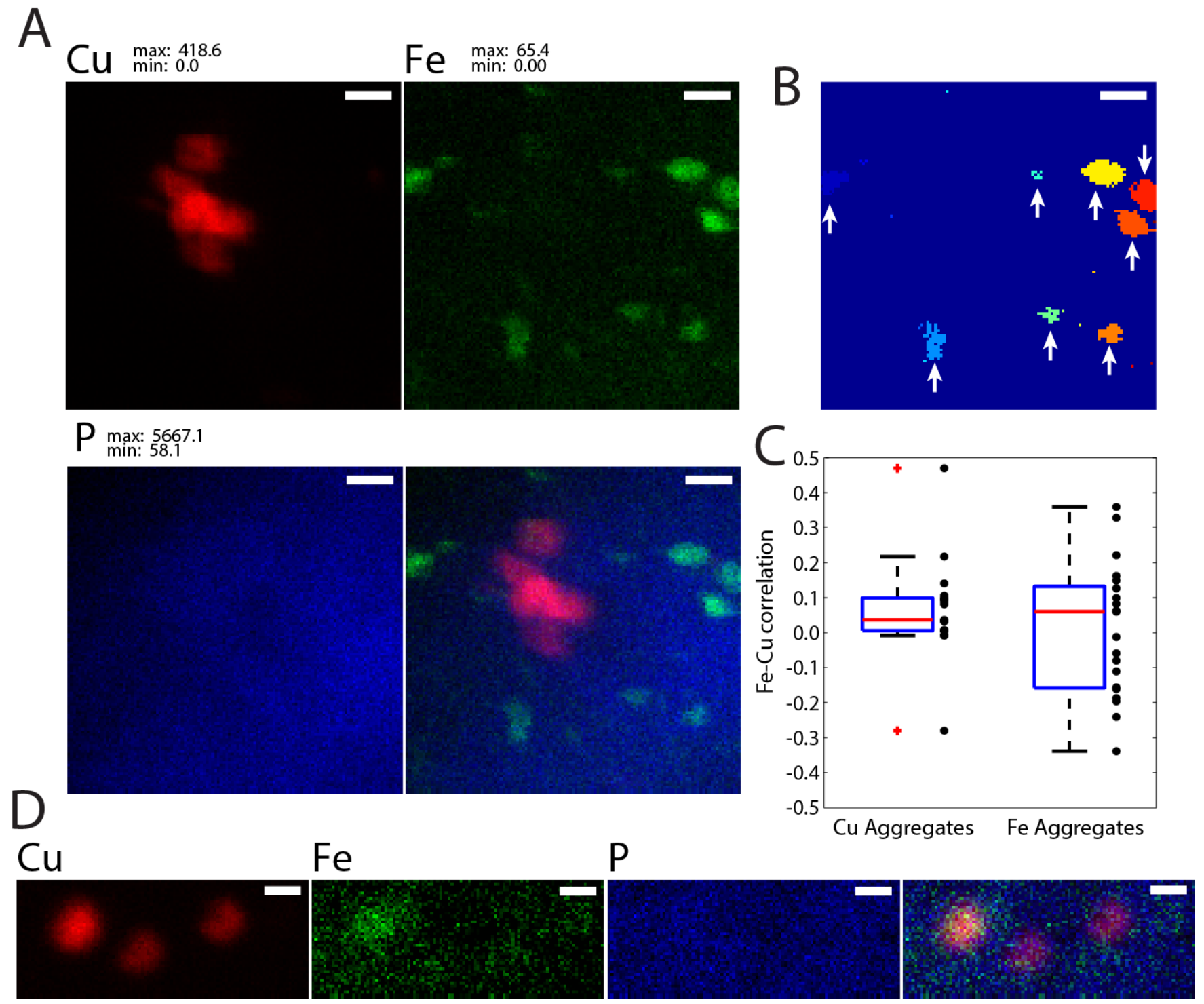

Figure 5. A. XRF map of $\mathrm{Cu}$ (red), Fe (green), and $\mathrm{P}$ (blue) and their overlay with $30 \mathrm{~nm}$ resolution from an unstained rat section. In untreated sections, $\mathrm{Cu}$ and $\mathrm{Fe}$ aggregates tend not co-localize. Scale bar: $500 \mathrm{~nm}$ B. Demonstration of particle detection on the Fe map in A. Clusters with arrows, which have 5 or more pixels, are used for co-localization analysis. Scale bar: $500 \mathrm{~nm}$. C. Co-localization statistics showing Pearson's correlation coefficient for Cu-rich clusters (left) and Fe-rich clusters (right). A boxplot showing the median, $25^{\text {th }}$ percentile, $75^{\text {th }}$ percentile and extremal values is given along with a scatter plot for individual clusters imaged with $30 \mathrm{~nm}$ or $40 \mathrm{~nm}$ resolution is shown. D. Co-localization of $\mathrm{Cu}, \mathrm{Fe}$, and $\mathrm{P}$ with $40 \mathrm{~nm}$ resolution demonstrating a case of $\mathrm{Fe}-\mathrm{Cu}$ colocalization in the left cluster $(\mathrm{r}=0.49)$. Display ranges are given in $\mathrm{mM}$ through a $5 \mu \mathrm{m}$ thick section. 
Table 1: Rodents and figures

\begin{tabular}{|c|c|c|c|c|c|}
\hline & $\begin{array}{l}\text { 129S1/SvImJ } \\
\text { Mouse }\end{array}$ & $\mathrm{C57BL} / 6 J^{\dagger}$ mouse & $\begin{array}{l}\text { C57BL/6J×SvImJ } \\
\text { mouse }\end{array}$ & CD-1 mouse & $\begin{array}{l}\text { Sprague- } \\
\text { Dawley rat }\end{array}$ \\
\hline $\begin{array}{l}\text { Number of } \\
\text { Rodents }\end{array}$ & 3 (9 weeks) & $\begin{array}{l}6 \text { (16 week), } \\
2 \text { wild-type ( } 28 \\
\text { week), } \\
2 \text { transgenic ( } 28 \\
\text { week) }\end{array}$ & 3 (ZnT3 weeks) & $\begin{array}{l}3 \text { (12 weeks), } \\
6 \text { (32 weeks) }\end{array}$ & 6 (12 weeks) \\
\hline Habenula & $\mathrm{Cu}+$ & $\mathrm{Cu}+{ }^{k}, \mathrm{DAB}+$ & Not Imaged & $\mathrm{DAB}+{ }^{\mathrm{f}}$ & $\mathrm{DAB}+{ }^{\mathrm{e}, 1}$ \\
\hline Hippocampus & $\mathrm{Cu}-$ & DAB- & $\mathrm{Cu}-{ }^{h}$ & DAB-, Cu- ${ }^{\mathrm{g}}$ & $\mathrm{DAB}+, \mathrm{Cu}+{ }^{\mathrm{i}}$ \\
\hline Hypothalamus & $\mathrm{DAB}+$ & $\mathrm{DAB}+, \mathrm{Cu}+$ & Not Imaged & $\mathrm{DAB}+{ }^{\mathrm{d}}, \mathrm{Cu}+$ & $\mathrm{DAB}+{ }^{\mathrm{c}}, \mathrm{Cu}+$ \\
\hline SVZ & $\mathrm{Cu}+{ }^{\mathrm{j}}$ & Not Imaged & $\mathrm{Cu}+$ & $\mathrm{Cu}+{ }^{b}$ & $\mathrm{Cu}+{ }^{\mathrm{a}}$ \\
\hline RMS & No Imaged & $\mathrm{Cu}-$ & Not Imaged & $\mathrm{Cu}^{\mathrm{b}}$ & $\mathrm{Cu}+{ }^{\mathrm{a}}$ \\
\hline
\end{tabular}

${ }^{\mathrm{a}}$ Figure $1 \mathrm{~A}, \mathrm{C} ;{ }^{\mathrm{b}}$ Figure 1B,D; Figure $4 \mathrm{C}, \mathrm{D}, \mathrm{E}{ }^{\mathrm{c}}$ Figure $3 \mathrm{~B} ;{ }^{\mathrm{d}}$ Figure $3 \mathrm{C} ;{ }^{\mathrm{e}}$ Figure $3 \mathrm{E} ;{ }^{\mathrm{f}}$ Figure $3 \mathrm{D} ;{ }^{\mathrm{g}}$ Figure 2 A; ${ }^{\mathrm{h}}$ Figure 2 B; ${ }^{\mathrm{i}}$ Figure $2 \mathrm{C}, \mathrm{D} ;{ }^{\mathrm{j}}$ Figure $5 ;{ }^{\mathrm{k}}$ Figure $4 \mathrm{~F} ;{ }^{1}$ Figure $4 \mathrm{G}$;

${ }^{\dagger}$ Both wild-type and transgenic overexpression of amyloid precursor protein and presinilin-1 
Table 2: $\mathrm{Cu}$ concentrations for 12 -week old rats and mice ( $\mathrm{mM}$ in $\mathbf{3 0} \mu \mathrm{m}$ thick section)

\begin{tabular}{|l|l|l|}
\hline & Sprague-Dawley Rats $(n=5)$ & CD-1 mice $(n=3)$ \\
\hline SVZ & $4.45 \pm 1.03[15]$ & $12.70 \pm 0.63$ \\
\hline RMS & $4.06 \pm 1.05[15]$ & $4.64 \pm 0.31$ \\
\hline Striatum & $3.27 \pm 0.79$ & $5.61 \pm 1.10$ \\
\hline Cortex & $3.12 \pm 0.70$ & $4.10 \pm 0.10$ \\
\hline
\end{tabular}

